\title{
SOSTENIBILIDAD Y RESILIENCIA EN LOS AGROSISTEMAS: IDENTIFICACIÓN DE CRITERIOS E INDICADORES (PÓSTER)
}

\author{
$\underline{\text { Luzvenia Miranda }}^{\mathrm{a}^{*}}$ y Inmaculada Marqués ${ }^{\mathrm{a}}$ \\ aDepartamento de Economía y Ciencias Sociales, Universidad Politécnica de Valéncia, (Valencia, \\ luzveniamp@gmail.com, imarques@esp.upv.es)
}

\section{Resumen}

En los últimos años han surgido debates en torno a las consecuencias de la crisis ambiental, entre ellas la preocupación por los efectos negativos de la agricultura en la producción de alimentos, las consecuencias del cambio climático y demás presiones socio-económicas. En este contexto surge la necesidad de identificar los criterios e indicadores en torno a la sostenibilidad y resiliencia de los agrosistemas.

Tradicionalmente la sostenibilidad agrícola se había enfocado al desarrollo económico sin considerar los efectos socioculturales y ambientales. La consideración conjunta de los aspectos económicos, socioculturales y ambientales genera una visión de mayor amplitud multidimensional vinculada a los capitales natural, producido, social y humano. Esta visión de la sostenibilidad integral, incorpora una visión de una agricultura capaz de afrontar eventos externos y con características resilientes, que incremente su capacidad de respuesta frente a adversidades. A pesar de que conceptualmente sostenibilidad y resiliencia se hayan considerado como terminos independientes y/o complementarios, en la funcionalidad de los agrosistemas se habrian utilizado indistintamente.

Se ha utilizado una metodológia descriptiva para identificar los criterios e indicadores que conducen al comportamiento sostenible-resiliente de agrosistemas desde una visión multidimensional con capacidad de resistencia, adaptación y recuperación frente a efectos del cambio climático, crisis económicas y sanitarias.

Palabras clave: Sostenibilidad, resiliencia, agrosistema, dimensiones, sistema productivo

\section{Introducción}

Según Astier et al. (2011) la percepción general de la sostenibilidad y resiliencia ha cobrado importancia por consecuencia de los impactos generados por la agricultura moderna afectando negativamente a los sistemas agrarios que tradicionalmente habian mostrado un equilibrio con el medio ambiente y con la administración de los recursos productivos. Surgiendo asi la necesidad de reforzar los aspectos que le confieren a los agrosistemas un carácter más sostenible y resiliente en función de las condiciones geográficas y agroclimaticas. En este contexto, las características que harian de un sistema productivo de una región sostenible resiliente, no necesariamente coincidirían con aquellos agrosistemas de otras regiones. Además los aspectos socioculturales, políticos y económicos habrian influido en el manejo y conservación de las explotaciones tradicionales de mayor sostenibilidad y resiliencia. Por lo que, como respuesta a estas condiciones y aspectos, han surgido metodologías de evaluación desde distintas percepciones y dimensiones.

El presente articulo, tiene por objetivo aclarar la correlación conceptual entre sostenibilidad y resiliencia e identificar algunos criterios e indicadores relacionados a los bienes y servicios que ofrecen los distintos agrosistemas, vinculados a sus capitales desde la dimensión económica, social y ambiental. Através de una metodología descriptiva y explicativa basada en una revisisón bibliográfica, se han analizado las variables cualitativas y cuantitativas de caracteres que son claves en el planteamiento de una guia metodologíca para la evaluación de los agrosistemas.

\section{Metodología}

El presente trabajo ha utilizado una metodología descriptiva y explicativa de las principales características que se deben considerar en la evaluación de los agrosistemas sostenible-resilientes. A partir de una revisión bibliográfica realizada en las bases de datos: Web of Science (WoS), Scopus y Sciencedirect para publicaciones sujetos a revisión de pares. Se ha utilizado los descriptores de búsqueda: "agroecosystem", seguido de un primer refine "sustainability" y un segundo refine con "resilience" y no se ha especificado el año, país o área temática. Posteriormente se ha deslindado las definiciones conceptuales entre sostenibilidad y resiliencia y se han descrito los cuatro capitales relacionados a la funcionalidad de los agrosistemas en sus distintas dimensiones. Se pretende en este trabajo responder las cuestiones: ¿Cuáles son los criterios e indicadores que se deben considerar en el análisis de los agrosistemas para determinar su naturaleza sostenible-resiliente? y ¿Cómo se pueden clasificar estas características desde un enfoque multidimensional? 


\section{Resultados}

Los resultados de la búsqueda bibliográfica se detallan en el Cuadro 1. De las 805 publicaciones provenientes de las tres bases de datos, a partir del resumen se han seleccionado 84 publicaciones relacionadas a enfoques conceptuales de sostenibilidad o resiliencia en agroecosistemas, seguidamente se han descartado los duplicados y finalmente nos ha quedado 62 publicaciones.

Cuadro 1. Resultados de la busqueda

\begin{tabular}{|c|c|c|c|c|}
\hline $\begin{array}{c}\text { Bases } \\
\text { de } \\
\text { datos }\end{array}$ & $\begin{array}{c}\text { Palabras clave y algoritmo de } \\
\text { búsqueda }\end{array}$ & $\begin{array}{c}\mathbf{N}^{\circ} \text { de } \\
\text { publicac } \\
\text { iones }\end{array}$ & $\begin{array}{c}\text { Selección } \\
\text { según } \\
\text { resumen }\end{array}$ & $\begin{array}{c}\text { Excluir } \\
\text { duplicad } \\
\text { os }\end{array}$ \\
\hline $\begin{array}{c}\text { Web of } \\
\text { science }\end{array}$ & $\begin{array}{c}\text { agroecosystem (4077) refinado } \\
\text { sustainability (481) refinado resilience }\end{array}$ & $\mathrm{n}=46$ & 17 & 0 \\
\hline Scopus & $\begin{array}{c}\text { agroecosystem (10013) refinado } \\
\text { sustainability (3031) refinado resilience }\end{array}$ & $\mathrm{n}=632$ & 57 & $13(44)$ \\
\hline $\begin{array}{c}\text { Science } \\
\text { direct }\end{array}$ & agroecosystem, sustainability, resilience & $\mathrm{n}=127$ & 10 & $2(8)$ \\
\hline & & 805 & 84 & Total 62 \\
\hline
\end{tabular}

Fuente: Elaboración propia

\section{Agrosistemas sostenibles y resilientes}

Primero, bajo el enfoque conceptual se ha tratado de ver las diferencias o similitudes entre agrosistema y agroecosistema. Grenón \& Mansilla (2008), indican que "los agrosistemas abarcan un conjunto de sistemas concretos que van desde el nivel fitosfera pasando por la zoosfera, el agroecosistema, la empresa agropecuaria, la cadena agroalimentaria y la región agroeconómica" y para su factibilidad requiere la integración y la capacidad del agroecosistema para mantener niveles de producción aceptables involucrados a la sostenibilidad. Esta definición da a entender al agrosistema como un sistema complejo que incluye al agroecosistema. Sin embargo, Jacques Tassin (2012); investigador en ecología vegetal, diferencia los ecosistemas de los agroecosistemas principalmente porque estos son manejados por el hombre, además indica que la definición de agroecología de Gliessman (2000) es un concepto holístico, que trata de aplicar conceptos y principios de la ecología al diseño y manejo de agroecosistemas sostenibles, determinando que el término agroecosistema es una simplificación o un atajo semántico para designar un agrosistema. Igualmente para Wojtkowski (2019) el agrosistema es un componente central de la agroecología aplicada, porque los sistemas agrarios estan compuestos por una o más especies de plantas y que a través de sus interacciones, muestran una ecología de comunidades que tienen por objetivo alcanzar el rendimiento preestablecido, reducir el riesgo y otros fines expresados económicamente. Por lo que se podria considerar que algunos autores coinciden que agrosistemas o agroecosistemas son conceptos similares.

Según Rosa (2018), el termino desarrollo sustentable llega al ámbito político en 1987 en el Informe de la Comisión Brundtland como objetivo para abordar los problemas de la crisis ambiental por los efectos de las acciones presentes sobre las generaciones futuras, dando énfasis a la relación entre el desarrollo económico y el aprovechamiento razonable del capital natural. En los últimos años, la sostenibilidad ha cobrado importancia en diversos campos de la investigación. Para Altieri y Nicholls (2000), bajo la base agroecológica tiene como finalidad el rendimiento sostenido, como la producción de alimentos de manera equilibrada en el tiempo y espacio, pese a las limitaciones ambientales o presiones socioeconómicas que pueden generarse. Sarandón \& Flores (2014) indican que los agroecosistemas sostenibles son sistemas multidimensionales, economicamente viables por la eficiencia del uso de los recursos, ecológicamente adecuados por la convervación del capital natural y socialmente aceptables por el respeto a las tradiciones y costumbres de la comunidad.

Por otro lado Peterson et al. (2018) indica que el concepto de resiliencia, fue utilizado por primera vez por el ecólogo CS Holling (1973) como la capacidad de los sistemas naturales de mantener su función y organización original. Pero el concepto ha evolucionado de la perspectiva ecológica al análisis de sistemas complejos integrados de personas y naturaleza "agroecosistemas". En este sentido Córdoba et al. (2020), indican que los agrosistemas al ser dirigidos por el hombre se adaptan según intereses o valores y no permiten la determinación de una estructura y función como sistema único sino de poder transformarse e innovar. Por lo que la resiliencia en los agrosistemas estaría dado de acuerdo a la planificación de diferentes grupos de poder con mayor o menor acceso a recursos.

Al integrar las perspectivas de sostenibilidad y resiliencia al agrosistema podemos ver la complejidad del sistema, como interdisciplinaria e integradora de innovación y adaptación. Para Bruckmeier y Pires (2018), 
se adopta el enfoque de resiliencia como un proceso adaptativo (capacidad de respuesta ante perturbaciones) y la sostenibilidad como un proceso transformador (equilibrio de los componentes del sistema social y ecológico) sumando a estos conceptos el riesgo y la vulnerabilidad. Según Zabala et al. (2021), la resiliencia es una pieza clave para asegurar la sostenibilidad a largo plazo de los agroecosistemas.

\section{Identificación de criterios e indicadores}

Tonolli \& Ferrer Gonzalez (2018) indican que para evaluar la sostenibilidad y resiliencia del agrosistema se han dado diferentes propuestas e investigaciones y la mayoría apunta a evaluar como un sistema integral, a través de indicadores y dimensiones. También Jules Pretty (2008) afirma que los agrosistemas dependen del flujo de valor de los servicios de activos que los influyen y controlan (desde la producción hasta el consumo) y desde esta perspectiva nos ayuda a responder cuestiones sobre el uso y mejor funcionamiento del agrosistema. Este valor está representado entre otros por el capital producido (activos económicos), natural (riqueza de los recursos naturales), social (cultura, redes, seguridad alimentaria) y humano (conocimiento, empleo y salud). Así mismo, para Sarandón (2002), la interacción de capitales es necesaria para la sustentabilidad, porque cada capital aumenta la productividad de otras formas de capital haciéndolas más eficientes.

A continuación se recogen en el Cuadro 2, los criterios e indicadores para el análisis del carácter sostenibleresiliente de los agrosistemas. El capital producido contempla la dimensión económica y está determinado por tres criterios: la capacidad de incentivar el crecimiento económico, la distribución equilibrada de los beneficios y la gestión de riesgos económicos. El capital natural contempla la dimensión ecológica y ambiental, presenta criterios de conservación del capital natural, aprovechamiento equilibrado de los recursos y la mitigación de riesgos agroclimáticos. El capital social con la dimensión sociocultural, viene determinado por criterios de seguridad alimentaria, preservación de raíces endógenas y la dinámica poblacional. Finalmente el capital humano presenta tres dimensiones: salud con el criterio de evaluación de alimentación nutritiva y de calidad, el empleo con el criterio de mano de obra y salario y la dimensión conocimientos y saberes a lo largo de la cadena de valor agroalimentaria. Cada criterio se analiza a través de sus indicadores, como se observa en el siguiente cuadro. Adicionalmente es posible desagregar los distintos criterios en subcriterios y determinar para los mismos indicadores que permitan evaluar cada dimensión y capital de un agrosistema.

Cuadro 2. Criterios e indicadores

\begin{tabular}{|c|c|c|c|}
\hline Capital & Dimensión & Criterios & Indicadores \\
\hline \multirow{3}{*}{ Producido } & \multirow{3}{*}{ Económica } & $\begin{array}{l}\text { Capacidad para incentivar un } \\
\text { aumento razonable del } \\
\text { crecimiento económico }\end{array}$ & $\begin{array}{l}\text { Beneficios económicos, nivel tecnológico en } \\
\text { la producción, investigación y desarrollo }\end{array}$ \\
\hline & & $\begin{array}{l}\text { Contribuir a una distribución más } \\
\text { equilibrada de los costos y } \\
\text { beneficio }\end{array}$ & $\begin{array}{l}\text { Diversificación productiva, tipos de } \\
\text { transformación/procesamiento y organización } \\
\text { y tamaño de explotaciones agraria }\end{array}$ \\
\hline & & Gestión de riesgos económicos & $\begin{array}{l}\text { Riesgo productivos, crediticio, de mercado, de } \\
\text { impuestos }\end{array}$ \\
\hline \multirow{3}{*}{ Natural } & Ecológica & $\begin{array}{l}\text { Capacidad para mantener y } \\
\text { conservar el capital natural }\end{array}$ & Conservación in situ y ex situ \\
\hline & \multirow[t]{2}{*}{ Ambiental } & $\begin{array}{l}\text { Uso de los recursos renovables y } \\
\text { no renovables sin exceder su ratio } \\
\text { de regeneración y su sustituto }\end{array}$ & $\begin{array}{l}\text { Prácticas de conservación de recursos } \\
\text { hídricos, contribución a la conservación y } \\
\text { purificación del aire atmosférico a nivel } \\
\text { regional y global }\end{array}$ \\
\hline & & Mitigar riesgos agroclimáticos & $\begin{array}{l}\text { Acciones para hacer frente a riesgos } \\
\text { climáticos, plagas enfermedades y uso de } \\
\text { productos }\end{array}$ \\
\hline \multirow{3}{*}{ Social } & \multirow{3}{*}{ Sociocultural } & $\begin{array}{l}\text { Seguridad alimentaria y acceso a } \\
\text { bienes y servicios básicos }\end{array}$ & $\begin{array}{l}\text { Capacidades del agrosistema para garantizar } \\
\text { la disponibilidad de alimentos de calidad para } \\
\text { consumo humano y condiciones de bienestar } \\
\text { de las comunidades rurales }\end{array}$ \\
\hline & & $\begin{array}{l}\text { Preservación de raíces endógenas } \\
\text { y participación comunal }\end{array}$ & $\begin{array}{l}\text { Participación de actores sociales en toma de } \\
\text { decisiones y preservación de conocimientos } \\
\text { ancestral }\end{array}$ \\
\hline & & $\begin{array}{l}\text { Dinámica poblacional y cohesión } \\
\text { territorial y configuración urbano } \\
\text { rural más equilibrada }\end{array}$ & $\begin{array}{l}\text { Indicadores demográficos, diseño y } \\
\text { planificación urbana y rural, organización } \\
\text { territorial y tenencia de tierras }\end{array}$ \\
\hline Humano & Salud & Repercusión en la salud & $\begin{array}{l}\text { Acciones de consumo adecuado de alimentos } \\
\text { saludables de dieta balanceada }\end{array}$ \\
\hline
\end{tabular}




\begin{tabular}{|c|c|l|l|} 
& Empleo & $\begin{array}{l}\text { Empleo y condiciones de trabajo } \\
\text { (mano de obra y salario) }\end{array}$ & $\begin{array}{l}\text { Promoción de la formalidad de empleadores y } \\
\text { condiciones adecuadas de contratación laboral } \\
\text { y la regulación del salario agrario }\end{array}$ \\
\cline { 2 - 4 } & $\begin{array}{c}\text { Conocimient } \\
\text { os }\end{array}$ & $\begin{array}{l}\text { Saberes y conocimiento a lo largo } \\
\text { de la cadena de valor } \\
\text { agroalimentaria }\end{array}$ & $\begin{array}{l}\text { Grado de instrucción del agricultor, presencia } \\
\text { de técnicos o especialistas agrarios }\end{array}$ \\
\hline
\end{tabular}

Fuente: Elaboración propia

Tomando en cuenta las consideraciones anteriores, al priorizar los criterios y funciones que cumplen cada uno de los capitales, se podría determinar el grado o nivel de sostenibilidad y resiliencia presente en el agrosistema.

\section{Conclusiones}

A manera de conclusión podemos indicar que no existe un deslinde conceptual definitivo entre agrosistema y agroecosistema ya que algunos autores lo consideran conceptos similares y otros consideran que el agroecosistema está dentro del agrosistema y sus relaciones comunes se basan en la funcionalidad de un determinado espacio geográfico orientado a la sostenibilidad.

Las características de agrosistemas sostenibles y resilientes han servido como un proceso adaptativo y transformador en los distintos sistemas de producción, en su evolución se han encontrado con una serie de factores, haciéndolas complejas en el monitoreo y evaluación, teniendo la necesidad de considerar distintas dimensiones y un trabajo multidisciplinario.

Además el uso de criterios e indicadores en el análisis de los agrosistemas nos podría contribuir en la determinación del grado o nivel de sostenibilidad y resiliencia.

\section{Bibliografía}

Altieri, M. y C. Nicholls. 2000. Agroecología: Teoría y práctica para una agricultura sustentable. Primera. Mexico.

Astier, Marta, Erika N. Speelman, Santiago López-Ridaura, Omar R. Masera, y Carlos E. GonzalezEsquivel. 2011. «Sustainability indicators, alternative strategies and trade-offs in peasant agroecosystems: analysing 15 case studies from Latin America». International Journal of Agricultural Sustainability 9(3):409-22.

Bruckmeier, Karl y Iva Pires. 2018. «Innovation as Transformation: Integrating the Socio-ecological Perspectives of Resilience and Sustainability». Pp. 209-31 en Advances in Spatial Science.

Córdoba, Cindy, Catalina Triviño, y Javier Toro Calderón. 2020. «Agroecosystem resilience. A conceptual and methodological framework for evaluation». PLOS ONE 15(4):1-20.

Grenón, Daniel y Carla Mansilla. 2008. Agromática: Aplicaciones informáticas en la formación de Ingenieros Agrónomos Agromatics : Informatics Applications in Agricultural Engineer Education.

Peterson, Caitlin A., Valerie T. Eviner, y Amélie C. M. Gaudin. 2018. «Ways forward for resilience research in agroecosystems». Agricultural Systems 162:19-27.

Pretty, Jules. 2008. «Agricultural sustainability: Concepts, principles and evidence». Philosophical Transactions of the Royal Society B: Biological Sciences 363(1491):447-65.

Rosa, Manuela Pires. 2018. «Innovative Urban Paradigms for Sustainability and Resilience». Pp. 189-207 en Advances in Spatial Science.

Sarandón, Santiago J. 2002. Agroecología. El camino hacia una agricultura sustentable. La Plata: Ediciones Científicas Americanas.

Sarandón, Santiago J. y Claudia C. Flores. 2014. Agroecología: bases teóricas para el diseño y manejo de Agroecosistemas sustentables. La Plata. editado por C. C. Sarandón, S. J. y Flores. Buenos Aires.

Tassin, Jacques. 2012. «Un agrosystéme est-il un écosystème?» Cahiers Agricultures 21(1):57-63.

Tonolli, Alejandro Javier y César Sergio Ferrer Gonzalez. 2018. «Comparación de marcos de evaluación de agroecosistemas». Tropical and Subtropical Agroecosystems 21:487-504.

Wojtkowski, P. 2019. «Agroecosystem Design». en Agroecology. 
Zabala, José A., José M. Martínez-Paz, y Francisco Alcon. 2021. «A comprehensive approach for agroecosystem services and disservices valuation». Science of the Total Environment 768:144859. 